\title{
Servicios otorgados en unidades de la Secretaría de Salud, 1997*
}

E 1 Sistema de Información en E Salud para Población Abierta (SISPA) representa un avance en la medida en que pretende generar e integrar la información institucional; está estructurado por subsistemas, y éstos, a su vez, en módulos que responden a las necesidades de información de la Secretaría de Salud (SSA).

La información generada por el SISPA, entre otras cosas, proporciona datos sobre la oferta y la demanda de los servicios de salud que se prestan a la población, a partir de informes mensuales emitidos por cada unidad médica de la SSA. Ello permite evaluar la operación y conocer tanto la cobertura como el impacto de dichos servicios; de esta forma, también se logra apoyar a investiga- dores, así como a quienes toman decisiones, con elementos suficientes para el diagnóstico de salud en los diferentes ámbitos administrativos.

Los cuadros que se presentan a continuación están divididos en dos secciones y se refieren a la información anual del SISPA correspondiente a 1997, de la cual se incluye sólo una pequeña parte. La primera sección da cuenta, de manera general, de las atenciones que se otorgaron, es decir, de la consulta externa, de los servicios hospitalarios y de los servicios auxiliares de diagnóstico.

En la segunda sección se exponen tabulaciones sobre los servicios otorgados a diferentes grupos poblacionales; inicia con las atenciones al menor de cinco años, continúa con lo referente al cuidado de la salud materna y los servicios de planificación familiar, para concluir con los accidentes atendidos y las actividades de detección de diferentes padecimientos, especialmente en los adultos.

Para obtener mayor detalle de la información aquí presentada, se pueden consultar las publicaciones de la Dirección General de Estadística e Informática, especialmente el Anuario estadístico de la Secretaría de Salud y el Boletín Mensual del SISPA, así como las tabulaciones específicas que se generan para cada uno de los programas operativos de la SSA. La consulta se puede hacer en el Centro de Referencia e Información Estadística en Salud, Leibnitz 20, 5o. piso, colonia Nueva Anzures, C.P. 11590, México, D.F.

* Sección preparada por la Dirección General de Estadística e Informática de la Secretaría de Salud, México. 


\section{Consulta externa otorgada. Estados Unidos Mexicanos, 1997}

\begin{tabular}{|c|c|c|c|c|c|c|c|c|}
\hline \multirow[b]{2}{*}{ Entidad federativa } & \multirow[b]{2}{*}{ Total } & \multicolumn{3}{|c|}{ Enfermedades } & \multirow[b]{2}{*}{ Consulta a sanos } & \multirow[b]{2}{*}{ Planificación familiar } & \multirow[b]{2}{*}{ Salud bucal } & \multirow[b]{2}{*}{ Salud mental } \\
\hline & & Transmisibles & Crónico-degenerativas & Otras & & & & \\
\hline EUM & 45463595 & 10510049 & 2242959 & 13224355 & 10798146 & 4919062 & 3375343 & 393681 \\
\hline Aguascalientes & 467046 & 106752 & 32171 & 130768 & 101023 & 40065 & 48390 & 7877 \\
\hline Baja California & 471536 & 85362 & 34679 & 167995 & 76364 & 55661 & 41220 & 10255 \\
\hline Baja California Sur & 301902 & 63010 & 23345 & 85741 & 75235 & 30916 & 18629 & 5026 \\
\hline Campeche & 526421 & 161621 & 16076 & 169398 & 92947 & 51963 & 34416 & 0 \\
\hline Coahuila & 956356 & 252534 & 56528 & 301275 & 146178 & 102836 & 95502 & 1503 \\
\hline Colima & 372510 & 99113 & 21149 & 118313 & 66708 & 38340 & 25294 & 3593 \\
\hline Chiapas & 1646220 & 472240 & 50655 & 544482 & 238822 & 160881 & 164100 & 15040 \\
\hline Chihuahua & 679411 & 143662 & 67698 & 200772 & 136677 & 100022 & 30015 & 565 \\
\hline Distrito Federal & 3531749 & 686267 & 135770 & 1350187 & 665101 & 170541 & 382609 & 141274 \\
\hline Durango & 621393 & 152233 & 43005 & 178632 & 105514 & 96740 & 40354 & 4915 \\
\hline Guanajuato & 2021294 & 541730 & 77874 & 527614 & 562129 & 191534 & 107594 & 12819 \\
\hline Guerrero & 2358284 & 622653 & 78256 & 738691 & 480248 & 341357 & 91528 & 5551 \\
\hline Hidalgo & 1261766 & 365423 & 52236 & 372264 & 269456 & 99089 & 95500 & 7798 \\
\hline Jalisco & 2669726 & 454940 & 207092 & 775311 & 802877 & 257062 & 149275 & 23169 \\
\hline México & 6986914 & 1436303 & 180789 & 1197335 & 2357866 & 1324324 & 475882 & 14415 \\
\hline Michoacán & 1537486 & 322574 & 103020 & 495941 & 301641 & 161404 & 143045 & 9861 \\
\hline Morelos & 996617 & 298794 & 31073 & 360576 & 139453 & 85047 & 79327 & 2347 \\
\hline $\mathrm{N}$ ayarit & 705933 & 162626 & 51094 & 195685 & 150558 & 81797 & 61387 & 2786 \\
\hline Nuevo León & 1926851 & 375898 & 146953 & 557583 & 525410 & 146223 & 149971 & 24813 \\
\hline 0 axaca & 1615977 & 462600 & 67472 & 518916 & 296723 & 137380 & 124028 & 8858 \\
\hline Puebla & 1770748 & 390499 & 94950 & 458729 & 451822 & 162008 & 203619 & 9121 \\
\hline Q uerétaro & 648300 & 163402 & 24465 & 208122 & 147695 & 53572 & 49782 & 1262 \\
\hline Q uintana Roo & 443067 & 122524 & 15627 & 124008 & 107604 & 48213 & 25091 & 0 \\
\hline San Luis Potosí & 907435 & 167035 & 75428 & 205767 & 251014 & 103383 & 99633 & 5175 \\
\hline Sinaloa & 890106 & 181025 & 61266 & 328007 & 175652 & 82457 & 54159 & 7540 \\
\hline Sonora & 1087802 & 264450 & 69031 & 385414 & 195379 & 85922 & 54612 & 32994 \\
\hline Tabasco & 2329317 & 513411 & 74470 & 732966 & 635535 & 148743 & 211417 & 12775 \\
\hline Tamaulipas & 1052735 & 228441 & 106111 & 365238 & 189718 & 102342 & 53267 & 7618 \\
\hline Tlaxcala & 929148 & 239404 & 33086 & 257396 & 266063 & 75472 & 53056 & 4671 \\
\hline Veracruz & 2097806 & 570191 & 117630 & 661218 & 400574 & 218198 & 126893 & 3102 \\
\hline Yucatán & 777044 & 239788 & 34461 & 258376 & 140913 & 61297 & 36053 & 6156 \\
\hline Zacatecas & 874695 & 163544 & 59499 & 251635 & 245247 & 104273 & 49695 & 802 \\
\hline
\end{tabular}

EUM $=$ Estados Unidos Mexicanos

Fuente: Secretaría de Salud, Dirección General de Estadística e Informática, Sistema de Información en Salud para Población A bierta (SSA, D GEl, SISPA) 
Cuadro II

Servicios hospitalarios. Estados Unidos Mexicanos, 1997

\begin{tabular}{|c|c|c|c|c|c|c|c|c|}
\hline \multirow[b]{2}{*}{ Entidad federativa } & \multirow[b]{2}{*}{ Egresos } & \multirow[b]{2}{*}{$\begin{array}{c}\text { Días } \\
\text { paciente }\end{array}$} & \multirow[b]{2}{*}{$\begin{array}{c}\text { Días } \\
\text { estancia }\end{array}$} & \multirow[b]{2}{*}{$\begin{array}{l}\text { Intervenciones } \\
\text { quirúrgicas }\end{array}$} & \multirow[b]{2}{*}{$\begin{array}{l}\text { Porcentaje de } \\
\text { ocupación* }\end{array}$} & \multirow[b]{2}{*}{$\begin{array}{l}\text { Promedio de } \\
\text { días estancia* }\end{array}$} & \multicolumn{2}{|c|}{ Mortalidad hospitalaria } \\
\hline & & & & & & & Total & Ajustada \\
\hline EUM & 1073189 & 4807916 & 4130212 & 551075 & 66.98 & 4.15 & 26.60 & 15.29 \\
\hline Aguascalientes & 23648 & 98218 & 83374 & 9679 & 67.06 & 3.55 & 18.00 & 12.71 \\
\hline Baja California & 24029 & 99540 & 83515 & 12662 & 80.40 & 3.70 & 41.89 & 28.65 \\
\hline Baja C alifornia Sur & 11182 & 43798 & 43394 & 6065 & 58.50 & 4.34 & 26.83 & 16.49 \\
\hline Campeche & 11816 & 40674 & 36976 & 6864 & 71.87 & 3.23 & 24.73 & 12.05 \\
\hline Coahuila & 23873 & 65212 & 57427 & 10362 & 40.34 & 2.50 & 17.61 & 11.95 \\
\hline Colima & 12963 & 39760 & 38698 & 6994 & 55.45 & 2.99 & 17.56 & 9.28 \\
\hline Chiapas & 47436 & 170480 & 135765 & 26119 & 85.24 & 2.97 & 33.95 & 17.87 \\
\hline Chihuahua & 15570 & 61134 & 50076 & 6284 & 75.99 & 3.55 & 34.43 & 21.81 \\
\hline Distrito Federal & 38931 & 412567 & 360498 & 28179 & 73.76 & 9.35 & 10.65 & 3.66 \\
\hline Durango & 20932 & 99960 & 81771 & 9685 & 54.82 & 4.00 & 28.74 & 16.15 \\
\hline Guanajuato & 64470 & 223728 & 252327 & 38067 & 68.29 & 4.37 & 25.62 & 12.23 \\
\hline Guerrero & 42041 & 147123 & 128828 & 23607 & 67.28 & 3.34 & 32.99 & 19.00 \\
\hline Hidalgo & 29463 & 197814 & 177901 & 11622 & 81.56 & 7.01 & 31.05 & 16.69 \\
\hline Jalisco & 64792 & 150044 & 153073 & 35430 & 61.38 & 2.50 & 14.31 & 8.37 \\
\hline México & 131573 & 862817 & 653381 & 72138 & 59.59 & 5.23 & 29.44 & 18.81 \\
\hline Michoacán & 44854 & 191734 & 158084 & 18909 & 70.84 & 4.12 & 29.62 & 14.80 \\
\hline Morelos & 28108 & 104454 & 85993 & 14003 & 81.48 & 3.41 & 34.36 & 19.30 \\
\hline $\mathrm{N}$ ayarit & 17929 & 49811 & 43345 & 7310 & 84.39 & 2.61 & 21.80 & 14.35 \\
\hline N uevo León & 33048 & 146241 & 120427 & 19637 & 68.74 & 3.66 & 20.72 & 12.34 \\
\hline 0 axaca & 34780 & 166356 & 154534 & 15311 & 64.92 & 5.00 & 30.07 & 18.23 \\
\hline Puebla & 31868 & 221092 & 158408 & 16960 & 53.29 & 5.96 & 29.23 & 14.55 \\
\hline Q uerétaro & 23808 & 87325 & 67575 & 12521 & 70.88 & 2.95 & 39.67 & 23.53 \\
\hline Q uintana Roo & 12380 & 41376 & 37320 & 6140 & 63.36 & 3.15 & 20.42 & 13.35 \\
\hline San Luis Potosí & 21460 & 61780 & 61342 & 9661 & 69.03 & 2.96 & 19.85 & 9.83 \\
\hline Sinaloa & 30467 & 104378 & 88481 & 15819 & 70.45 & 3.25 & 31.38 & 17.69 \\
\hline Sonora & 46967 & 223263 & 193861 & 14972 & 62.61 & 4.26 & 21.23 & 13.68 \\
\hline Tabasco & 52560 & 210922 & 185336 & 31319 & 78.52 & 3.79 & 28.07 & 18.84 \\
\hline Tamaulipas & 48655 & 199264 & 184747 & 23037 & 69.30 & 3.84 & 29.63 & 15.39 \\
\hline Tlaxcala & 19462 & 50714 & 49305 & 9442 & 60.53 & 2.67 & 24.27 & 9.17 \\
\hline Veracruz & 23170 & 83595 & 73115 & 11133 & 83.19 & 3.81 & 33.42 & 16.41 \\
\hline Yucatán & 17402 & 86803 & 81721 & 8922 & 81.72 & 5.18 & 26.90 & 16.93 \\
\hline Zacatecas & 23552 & 65939 & 49614 & 12222 & 93.96 & 2.58 & 24.44 & 12.74 \\
\hline
\end{tabular}

EUM $=$ Estados Unidos Mexicanos

*Se refiere exclusivamente a unidades hospitalarias

Fuente: SSA, DGEI, SISPA 
Cuadro III

Servicios auxiliares de diagnóstico. Estados Unidos Mexicanos, 1997

\begin{tabular}{|c|c|c|c|c|c|c|c|c|c|c|}
\hline \multirow[b]{2}{*}{ Entidad federativa } & \multicolumn{2}{|c|}{ Análisis clínicos } & \multicolumn{2}{|c|}{ Rayos X } & \multicolumn{2}{|c|}{ Anatomía patológica } & \multicolumn{2}{|c|}{ Electrocardiograma } & \multicolumn{2}{|c|}{ Ultrasonido } \\
\hline & Estudios & Personas & Estudios & Personas & Estudios & Personas & Estudios & Personas & Estudios & Personas \\
\hline EUM & 21201333 & 5620075 & 1890750 & 1499967 & 252902 & 229821 & 121018 & 114367 & 207542 & 198811 \\
\hline Aguascalientes & 530410 & 118681 & 108260 & 85840 & 2948 & 2922 & 4059 & 3924 & 3567 & 3540 \\
\hline Baja California & 376610 & 92771 & 57211 & 37283 & 8018 & 5123 & 13666 & 9923 & 3165 & 2908 \\
\hline Baja C alifornia Sur & 229519 & 78352 & 25840 & 21908 & 15561 & 15561 & 1321 & 1211 & 4036 & 3985 \\
\hline Campeche & 252649 & 67919 & 15553 & 13836 & 1031 & 1006 & 616 & 616 & 4329 & 4316 \\
\hline Coahuila & 451244 & 136510 & 28693 & 23825 & 8075 & 8067 & 1972 & 1894 & 6830 & 6830 \\
\hline Colima & 181930 & 66124 & 22027 & 18478 & 1814 & 1814 & 455 & 455 & 2292 & 2292 \\
\hline Chiapas & 784056 & 162880 & 57191 & 40164 & 8265 & 2129 & 2506 & 2451 & 4541 & 4473 \\
\hline Chihuahua & 317714 & 100506 & 26975 & 22348 & 2140 & 2081 & 2126 & 1955 & 579 & 579 \\
\hline Distrito Federal & 2474833 & 487392 & 154917 & 131038 & 13048 & 12288 & 9698 & 9432 & 16928 & 16750 \\
\hline Durango & 330532 & 106754 & 42331 & 37012 & 2949 & 2940 & 4002 & 3724 & 7187 & 7175 \\
\hline Guanajuato & 650324 & 207138 & 84279 & 69066 & 21649 & 21647 & 1616 & 1473 & 6522 & 6432 \\
\hline Guerrero & 889978 & 170822 & 61642 & 45937 & 4131 & 4131 & 3443 & 2852 & 6895 & 6847 \\
\hline Hidalgo & 445919 & 135557 & 39799 & 33469 & 2570 & 2570 & 1021 & 1012 & 9011 & 8512 \\
\hline Jalisco & 1046700 & 309956 & 82672 & 63292 & 22624 & 22144 & 5765 & 5750 & 2908 & 2908 \\
\hline México & 2490370 & 644153 & 193080 & 149771 & 37529 & 36468 & 13059 & 12943 & 22607 & 22284 \\
\hline Michoacán & 600851 & 221405 & 69460 & 56609 & 5956 & 5956 & 4378 & 4373 & 10424 & 10245 \\
\hline Morelos & 447899 & 131566 & 48810 & 41708 & 4608 & 4588 & 4089 & 4086 & 5694 & 5307 \\
\hline $\mathrm{N}$ ayarit & 214731 & 90830 & 24566 & 16225 & 2437 & 765 & 1867 & 1671 & 2332 & 2332 \\
\hline Nuevo León & 813861 & 198270 & 99641 & 82976 & 5128 & 5128 & 6443 & 6369 & 978 & 975 \\
\hline 0 axaca & 406711 & 131597 & 57569 & 41262 & 5500 & 2330 & 4942 & 4783 & 3869 & 3869 \\
\hline Puebla & 647801 & 197289 & 60893 & 47810 & 7240 & 4080 & 3339 & 3239 & 6838 & 6665 \\
\hline Q uerétaro & 342213 & 77500 & 36253 & 31452 & 2217 & 2112 & 1254 & 1254 & 4004 & 3474 \\
\hline Q uintana Roo & 331054 & 127539 & 20423 & 17751 & 6337 & 6337 & 276 & 267 & 2983 & 2983 \\
\hline San Luis Potosí & 612719 & 125520 & 25382 & 22476 & 3321 & 3312 & 1882 & 1875 & 3745 & 3727 \\
\hline Sinaloa & 555809 & 159323 & 60040 & 48773 & 6460 & 6312 & 3676 & 3617 & 8288 & 8218 \\
\hline Sonora & 1132311 & 293018 & 102492 & 79722 & 4092 & 4082 & 2561 & 2517 & 2696 & 2696 \\
\hline Tabasco & 1153548 & 247024 & 79156 & 58685 & 7208 & 7206 & 3918 & 3918 & 15250 & 14032 \\
\hline Tamaulipas & 884012 & 263249 & 85926 & 66942 & 12084 & 11555 & 8317 & 8098 & 11778 & 9133 \\
\hline Tlaxcala & 278374 & 62769 & 32389 & 21531 & 4084 & 2070 & 970 & 970 & 5788 & 5188 \\
\hline Veracruz & 498070 & 206908 & 23740 & 17354 & 2354 & 1959 & 3222 & 3222 & 7968 & 6898 \\
\hline Yucatán & 437834 & 104881 & 26437 & 23117 & 17706 & 17706 & 3493 & 3476 & 7230 & 7230 \\
\hline Zacatecas & 390747 & 95872 & 37103 & 32307 & 3818 & 3432 & 1066 & 1017 & 6280 & 6008 \\
\hline
\end{tabular}

EUM $=$ Estados Unidos Mexicanos

Fuente: SSA, D GEI, SISPA 
Cuadro IV

Atención al menor de cinco años. Estados Unidos Mexicanos, 1997

\begin{tabular}{|c|c|c|c|c|c|c|c|c|}
\hline \multirow[b]{2}{*}{ Entidad federativa } & \multirow[b]{2}{*}{ Consulta a niño sano } & \multicolumn{3}{|c|}{ Consultas según presencia de desnutrición } & \multicolumn{2}{|c|}{$\begin{array}{l}\text { Consultas enfermedad } \\
\text { diarreica aguda }\end{array}$} & \multicolumn{2}{|c|}{$\begin{array}{l}\text { Consultas infección } \\
\text { respiratoria aguda }\end{array}$} \\
\hline & & Total & Sin desnutrición & Con desnutrición & Total & Primera vez & Total & Primera vez \\
\hline EUM & 2631277 & 8263351 & 7473403 & 789948 & 861682 & 757390 & 3362474 & 2674298 \\
\hline Aguascalientes & 17306 & 79570 & 73054 & 6516 & 7241 & 6512 & 42923 & 32295 \\
\hline Baja California & 11374 & 66610 & 64630 & 1980 & 9175 & 8267 & 35063 & 26654 \\
\hline Baja California Sur & 14592 & 46231 & 44067 & 2164 & 3850 & 3408 & 17478 & 13089 \\
\hline Campeche & 22966 & 118146 & 100656 & 17490 & 15254 & 13893 & 57721 & 45796 \\
\hline Coahuila & 19917 & 159031 & 149108 & 9923 & 18416 & 16544 & 89499 & 71234 \\
\hline Colima & 12768 & 69058 & 65091 & 3967 & 5964 & 4994 & 31845 & 23060 \\
\hline Chiapas & 35173 & 237442 & 198550 & 38892 & 62671 & 57520 & 97496 & 84634 \\
\hline Chihuahua & 25142 & 101490 & 93882 & 7608 & 13608 & 12364 & 53895 & 44670 \\
\hline Distrito Federal & 99695 & 412029 & 386797 & 25232 & 26653 & 22964 & 217071 & 184728 \\
\hline Durango & 17471 & 88981 & 83375 & 5606 & 12976 & 11336 & 54151 & 40654 \\
\hline Guanajuato & 96943 & 437270 & 399086 & 38184 & 44390 & 38927 & 239361 & 194362 \\
\hline Guerrero & 87140 & 404748 & 342273 & 62475 & 54743 & 48644 & 145785 & 119496 \\
\hline Hidalgo & 43844 & 201398 & 179168 & 22230 & 19501 & 17089 & 114332 & 93195 \\
\hline Jalico & 174908 & 473377 & 436666 & 36711 & 28512 & 25436 & 181829 & 149808 \\
\hline México & 858317 & 1721710 & 1598830 & 122880 & 154161 & 131308 & 587083 & 452749 \\
\hline Michoacán & 41414 & 219186 & 205387 & 13799 & 25702 & 23080 & 93969 & 80490 \\
\hline Morelos & 23929 & 164467 & 145081 & 19386 & 21852 & 18646 & 90527 & 71800 \\
\hline $\mathrm{N}$ ayarit & 33770 & 110077 & 101117 & 8960 & 11398 & 9802 & 42790 & 34154 \\
\hline Nuevo León & 156460 & 360018 & 351893 & 8125 & 16205 & 13906 & 117235 & 93513 \\
\hline 0 axaca & 66274 & 296029 & 229506 & 66523 & 48934 & 44502 & 107661 & 91836 \\
\hline Puebla & 97463 & 309210 & 268907 & 40303 & 33375 & 29032 & 106697 & 85718 \\
\hline Q uerétaro & 21180 & 119933 & 107666 & 12267 & 10477 & 9400 & 57211 & 46806 \\
\hline Q uintana Roo & 10554 & 59930 & 49413 & 10517 & 8095 & 7377 & 31572 & 25325 \\
\hline San Luis Potosí & 79111 & 187952 & 145283 & 42669 & 15335 & 13621 & 61779 & 45353 \\
\hline Sinaloa & 25261 & 138733 & 133391 & 5342 & 19637 & 17840 & 56615 & 45424 \\
\hline Sonora & 28438 & 202713 & 197820 & 4893 & 26367 & 22589 & 103065 & 67638 \\
\hline Tabasco & 253644 & 533166 & 496906 & 36260 & 39775 & 33965 & 119291 & 89115 \\
\hline Tamaulipas & 34048 & 150848 & 144166 & 6682 & 14044 & 12390 & 75128 & 56899 \\
\hline Tlaxcala & 68196 & 201190 & 179804 & 21386 & 12548 & 9484 & 74386 & 51193 \\
\hline Veracruz & 74514 & 345670 & 301337 & 44333 & 42611 & 39285 & 128536 & 105608 \\
\hline Yucatán & 27638 & 148986 & 116899 & 32087 & 22822 & 20124 & 73953 & 63074 \\
\hline Zacatecas & 51827 & 98152 & 83594 & 14558 & 15390 & 13141 & 56527 & 43928 \\
\hline
\end{tabular}

$\mathrm{EUM}=$ Estados Unidos Mexicanos

Fuente: SSA, DGEI, SISPA 


\section{Cuadro V}

\section{Atención materna. Estados Unidos Mexicanos, 1997}

\begin{tabular}{|c|c|c|c|c|c|c|c|c|}
\hline \multirow[b]{2}{*}{ Entidad federativa } & \multirow[b]{2}{*}{ Embarazadas atendidas } & \multirow[b]{2}{*}{ Consultas a embarazadas } & \multicolumn{3}{|c|}{$\mathrm{Nacimientos}$ atendidos } & \multicolumn{2}{|c|}{ Nacidos vivos } & \multirow[b]{2}{*}{ Abortos atendidos } \\
\hline & & & Total & Parto & Cesárea & Total & Con bajo peso & \\
\hline EUM & 1113981 & 3098833 & 454894 & 347458 & 107436 & 451828 & 35312 & 55761 \\
\hline Aguascalientes & 12642 & 42013 & 8485 & 6022 & 2463 & 8448 & 590 & 1097 \\
\hline Baja California & 13657 & 33275 & 10315 & 8323 & 1992 & 10337 & 765 & 1444 \\
\hline Baja C alifornia Sur & 6798 & 21251 & 3738 & 2855 & 883 & 3724 & 194 & 461 \\
\hline Campeche & 14749 & 41778 & 4977 & 3705 & 1272 & 4980 & 282 & 585 \\
\hline Coahuila & 22625 & 52956 & 9296 & 7021 & 2275 & 9265 & 568 & 1355 \\
\hline Colima & 8314 & 25482 & 4803 & 3611 & 1192 & 4775 & 293 & 656 \\
\hline Chiapas & 42338 & 87599 & 19194 & 13598 & 5596 & 18852 & 1690 & 2859 \\
\hline Chihuahua & 16357 & 38253 & 5180 & 4239 & 941 & 5158 & 351 & 664 \\
\hline Distrito Federal & 56606 & 141185 & 18153 & 11498 & 6655 & 18108 & 2168 & 2513 \\
\hline Durango & 18940 & 48795 & 7484 & 5654 & 1830 & 7434 & 608 & 1002 \\
\hline Guanajuato & 62947 & 179265 & 31580 & 24950 & 6630 & 31290 & 2435 & 3714 \\
\hline Guerrero & 54042 & 115918 & 19536 & 16111 & 3425 & 19440 & 1799 & 1987 \\
\hline Hidalgo & 31028 & 85552 & 12780 & 10049 & 2731 & 12657 & 1234 & 1592 \\
\hline Jalisco & 73821 & 309686 & 31823 & 25310 & 6513 & 31829 & 1556 & 3183 \\
\hline México & 198910 & 468083 & 63585 & 50271 & 13314 & 62864 & 5498 & 7684 \\
\hline Michoacán & 43513 & 107568 & 17453 & 13927 & 3526 & 17319 & 1156 & 1926 \\
\hline Morelos & 20273 & 54451 & 10016 & 7219 & 2797 & 9870 & 1063 & 1563 \\
\hline $\mathrm{N}$ ayarit & 14897 & 48980 & 6405 & 5004 & 1401 & 6676 & 265 & 804 \\
\hline N uevo León & 37724 & 130869 & 15027 & 9995 & 5032 & 14989 & 899 & 1802 \\
\hline 0 axaca & 37517 & 95576 & 14724 & 10736 & 3988 & 14516 & 1436 & 1803 \\
\hline Puebla & 42477 & 103764 & 13298 & 10454 & 2844 & 13057 & 1244 & 1969 \\
\hline Q uerétaro & 20280 & 56567 & 11003 & 7847 & 3156 & 10875 & 931 & 1239 \\
\hline Q uintana Roo & 11187 & 28831 & 4949 & 3638 & 1311 & 4921 & 393 & 833 \\
\hline San Luis Potosí & 22540 & 74226 & 11007 & 8910 & 2097 & 10915 & 907 & 1351 \\
\hline Sinaloa & 24498 & 64587 & 9657 & 7358 & 2299 & 9609 & 576 & 1404 \\
\hline Sonora & 23334 & 69804 & 15184 & 11953 & 3231 & 15202 & 846 & 1742 \\
\hline Tabasco & 45161 & 149644 & 18600 & 13469 & 5131 & 18469 & 1161 & 1945 \\
\hline Tamaulipas & 26770 & 68928 & 17769 & 12789 & 4980 & 17682 & 1288 & 2202 \\
\hline Tlaxcala & 16207 & 48864 & 8555 & 6380 & 2175 & 8457 & 934 & 1103 \\
\hline Veracruz & 56494 & 178911 & 12025 & 10145 & 1880 & 11963 & 533 & 1116 \\
\hline Yucatán & 18164 & 59801 & 7680 & 5492 & 2188 & 7601 & 870 & 816 \\
\hline Zacatecas & 19171 & 66371 & 10613 & 8925 & 1688 & 10546 & 779 & 1347 \\
\hline
\end{tabular}

EUM $=$ Estados Unidos Mexicanos

Fuente: SSA, D GEI, SISPA 


\section{Cuadro VI}

\section{Servicios de Planificación familiar. Estados Unidos Mexicanos, 1997}

\begin{tabular}{|c|c|c|c|c|c|c|c|}
\hline \multirow[b]{2}{*}{ Entidad federativa } & \multirow[b]{2}{*}{$\begin{array}{l}\text { Consultas } \\
\text { y atenciones }\end{array}$} & \multirow[b]{2}{*}{$\begin{array}{l}\text { Nuevas } \\
\text { aceptantes }\end{array}$} & \multirow[b]{2}{*}{$\begin{array}{l}\text { Usuarias } \\
\text { activas }\end{array}$} & \multicolumn{3}{|c|}{ Intervenciones quirúrgicas } & \multirow[b]{2}{*}{$\begin{array}{l}\text { Aceptantes post } \\
\text { evento obstétrico }\end{array}$} \\
\hline & & & & Total & $\begin{array}{l}\text { Oclusión } \\
\text { tubaria }\end{array}$ & Vasectomía & \\
\hline EUM & 8989199 & 867837 & 2346985 & 89208 & 83433 & 5775 & 174106 \\
\hline Aguascalientes & 125274 & 9943 & 21942 & 1355 & 1248 & 107 & 3171 \\
\hline Baja California & 103356 & 15150 & 24292 & 1556 & 1404 & 152 & 5226 \\
\hline Baja California Sur & 56671 & 5467 & 14764 & 716 & 690 & 26 & 1797 \\
\hline Campeche & 97871 & 10467 & 17880 & 1603 & 1542 & 61 & 1879 \\
\hline Coahuila & 222354 & 18157 & 41539 & 1732 & 1674 & 58 & 4395 \\
\hline Colima & 66690 & 9955 & 23393 & 972 & 930 & 42 & 2980 \\
\hline Chiapas & 337199 & 37458 & 90453 & 5441 & 5264 & 177 & 11319 \\
\hline Chihuahua & 172948 & 17442 & 44580 & 926 & 800 & 126 & 2531 \\
\hline Distrito Federal & 312734 & 59038 & 85294 & 5548 & 4374 & 1174 & 10166 \\
\hline Durango & 174518 & 16310 & 49454 & 1271 & 1196 & 75 & 1984 \\
\hline Guanajuato & 365406 & 39536 & 85580 & 3928 & 3821 & 107 & 5860 \\
\hline Guerrero & 534340 & 39030 & 146710 & 7083 & 6828 & 255 & 5489 \\
\hline Hidalgo & 203945 & 21550 & 61535 & 2966 & 2797 & 169 & 4156 \\
\hline Jalisco & 490545 & 52887 & 139380 & 4366 & 4044 & 322 & 8904 \\
\hline México & 1735865 & 117152 & 490083 & 8908 & 8583 & 325 & 28173 \\
\hline Michoacán & 393228 & 42600 & 104241 & 3489 & 3122 & 367 & 6758 \\
\hline Morelos & 188894 & 15845 & 43878 & 2209 & 2023 & 186 & 4223 \\
\hline $\mathrm{N}$ ayarit & 180205 & 16890 & 37100 & 1227 & 1183 & 44 & 2352 \\
\hline Nuevo León & 271275 & 40782 & 81888 & 2678 & 2564 & 114 & 9288 \\
\hline 0 axaca & 257248 & 22851 & 70995 & 3059 & 2947 & 112 & 5991 \\
\hline Puebla & 436738 & 53985 & 128467 & 5667 & 5220 & 447 & 7384 \\
\hline Querétaro & 113256 & 10902 & 36314 & 1836 & 1745 & 91 & 2175 \\
\hline Q uintana Roo & 82214 & 7536 & 14581 & 1239 & 1036 & 203 & 1107 \\
\hline San Luis Potosí & 178558 & 20336 & 51159 & 1724 & 1597 & 127 & 4122 \\
\hline Sinaloa & 195791 & 21549 & 42677 & 1731 & 1574 & 157 & 1829 \\
\hline Sonora & 208072 & 14876 & 38117 & 1974 & 1752 & 222 & 3799 \\
\hline Tabasco & 282892 & 22054 & 90686 & 4517 & 4430 & 87 & 8719 \\
\hline Tamaulipas & 270987 & 19365 & 34355 & 1750 & 1606 & 144 & 7266 \\
\hline Tlaxcala & 125537 & 14113 & 39963 & 2290 & 2194 & 96 & 3755 \\
\hline Veracruz & 498360 & 43890 & 108413 & 2738 & 2616 & 122 & 2272 \\
\hline Yucatán & 138062 & 9841 & 31917 & 1464 & 1407 & 57 & 2517 \\
\hline Zacatecas & 168166 & 20880 & 55355 & 1245 & 1222 & 23 & 2519 \\
\hline
\end{tabular}

EUM $=$ Estados Unidos Mexicanos

Fuente: SSA, DGEI, SISPA 


\section{Accidentes atendidos. Estados Unidos Mexicanos, 1997}

\begin{tabular}{|c|c|c|c|c|c|c|c|}
\hline Entidad federativa & Total & $<5$ años & De 5 a 14 años & De 15 a 24 años & De 25 a 49 años & De 50 a 64 años & De 65 y más años \\
\hline EUM & 787477 & 94901 & 197505 & 186024 & 208907 & 61836 & 38304 \\
\hline Aguascalientes & 14685 & 2201 & 3880 & 3577 & 3320 & 981 & 726 \\
\hline Baja California & 15653 & 1720 & 3037 & 3677 & 5505 & 1164 & 550 \\
\hline Baja California Sur & 4415 & 516 & 950 & 1148 & 1455 & 217 & 129 \\
\hline Campeche & 8970 & 865 & 2000 & 2382 & 2790 & 597 & 336 \\
\hline Coahuila & 17517 & 2320 & 4313 & 4220 & 4499 & 1304 & 861 \\
\hline Colima & 13071 & 1240 & 3422 & 3270 & 3407 & 1066 & 666 \\
\hline Chiapas & 31262 & 3202 & 6550 & 8284 & 9370 & 2728 & 1128 \\
\hline Chihuahua & 16818 & 2102 & 4388 & 3655 & 4484 & 1416 & 773 \\
\hline Distrito Federal & 34628 & 3988 & 8722 & 7980 & 9348 & 2814 & 1776 \\
\hline Durango & 8292 & 1041 & 2386 & 1838 & 2061 & 624 & 342 \\
\hline Guanajuato & 57456 & 7165 & 14271 & 14887 & 13544 & 4474 & 3115 \\
\hline Guerrero & 50855 & 5641 & 13278 & 11458 & 13365 & 4328 & 2785 \\
\hline Hidalgo & 24726 & 2264 & 6457 & 5354 & 6843 & 2389 & 1419 \\
\hline Jalisco & 32289 & 3858 & 8884 & 7694 & 7759 & 2321 & 1773 \\
\hline México & 99212 & 13763 & 26018 & 23157 & 25762 & 6576 & 3936 \\
\hline Michoacán & 23886 & 3257 & 6781 & 5306 & 5602 & 1641 & 1299 \\
\hline Morelos & 35886 & 4779 & 9084 & 8564 & 9263 & 2493 & 1703 \\
\hline $\mathrm{N}$ ayarit & 14651 & 1874 & 3500 & 3192 & 3502 & 1422 & 1161 \\
\hline Nuevo León & 19485 & 3424 & 5240 & 4149 & 4584 & 1234 & 854 \\
\hline 0 axaca & 38644 & 3474 & 9635 & 8765 & 10979 & 3586 & 2205 \\
\hline Puebla & 25479 & 2456 & 6565 & 6084 & 6836 & 2224 & 1314 \\
\hline Q uerétaro & 12941 & 1400 & 3412 & 3192 & 3449 & 861 & 627 \\
\hline Q uintana Roo & 11908 & 1255 & 2776 & 3323 & 3601 & 654 & 299 \\
\hline San Luis Potosí & 14496 & 1764 & 4137 & 3070 & 3504 & 1161 & 860 \\
\hline Sinaloa & 13802 & 1684 & 3643 & 2972 & 3666 & 1065 & 772 \\
\hline Sonora & 31669 & 4432 & 7285 & 6811 & 8519 & 3154 & 1468 \\
\hline Tabasco & 30010 & 3690 & 6236 & 8157 & 8783 & 2144 & 1000 \\
\hline Tamaulipas & 18844 & 2875 & 4726 & 4497 & 4707 & 1326 & 713 \\
\hline Tlaxcala & 14800 & 1767 & 3327 & 3710 & 4045 & 1218 & 733 \\
\hline Veracruz & 26848 & 2431 & 7194 & 6068 & 7579 & 2328 & 1248 \\
\hline Yucatán & 12449 & 1105 & 2529 & 2942 & 3714 & 1236 & 923 \\
\hline Zacatecas & 11830 & 1348 & 2879 & 2641 & 3062 & 1090 & 810 \\
\hline
\end{tabular}

$\mathrm{EUM}=$ Estados Unidos Mexicanos

Fuente: SSA, D GEI, SISPA 
Cuadro VIII

Detección de enfermedades. Estados Unidos Mexicanos, 1997

\begin{tabular}{|c|c|c|c|c|c|c|c|c|}
\hline \multirow[b]{2}{*}{ Entidad federativa } & \multirow[b]{2}{*}{ Cáncer cervicouterino } & \multirow[b]{2}{*}{ Cáncer de mama } & \multicolumn{2}{|c|}{ Diabetes mellitus } & \multicolumn{2}{|c|}{ Hipertensión arterial } & \multicolumn{2}{|c|}{ Tuberculosis } \\
\hline & & & Hombres & M ujeres & Hombres & M ujeres & Hombres & M ujeres \\
\hline EUM & 1460727 & 2466714 & 828173 & 1887814 & 2513751 & 6212502 & 167171 & 211425 \\
\hline
\end{tabular}

\begin{tabular}{|c|c|c|c|c|c|c|c|c|}
\hline A guascalientes & 20124 & 25116 & 8038 & 23986 & 18126 & 54967 & 963 & 997 \\
\hline Baja California & 20882 & 20380 & 13753 & 29772 & 34287 & 78241 & 4090 & 3753 \\
\hline Baja California Sur & 13714 & 14011 & 5231 & 9501 & 13082 & 28884 & 1898 & 1372 \\
\hline Campeche & 19625 & 27919 & 10474 & 22710 & 22291 & 46680 & 1091 & 1632 \\
\hline Coahuila & 27378 & 37559 & 25847 & 55539 & 64738 & 154004 & 2991 & 4210 \\
\hline Colima & 17197 & 22987 & 6356 & 10765 & 21767 & 41888 & 2295 & 3114 \\
\hline Chiapas & 36535 & 88893 & 46818 & 81047 & 150801 & 305572 & 7154 & 7624 \\
\hline Chihuahua & 29773 & 47445 & 32742 & 62249 & 59548 & 128580 & 4552 & 4988 \\
\hline Distrito Federal & 127947 & 133831 & 34194 & 107573 & 226793 & 457105 & 3286 & 6522 \\
\hline Durango & 28318 & 50620 & 16961 & 47400 & 52404 & 147843 & 5303 & 6620 \\
\hline Guanajuato & 61774 & 117740 & 26070 & 63970 & 112800 & 307072 & 4376 & 5617 \\
\hline Guerrero & 99535 & 118564 & 49587 & 92959 & 155316 & 317255 & 9880 & 12010 \\
\hline Hidalgo & 38614 & 80988 & 35731 & 74211 & 104493 & 250316 & 6723 & 9146 \\
\hline Jalisco & 102052 & 98473 & 22962 & 54894 & 79421 & 217675 & 9638 & 11937 \\
\hline México & 98066 & 296484 & 53364 & 119023 & 256586 & 655518 & 4620 & 7865 \\
\hline Michoacán & 31876 & 71939 & 34609 & 82640 & 84136 & 221227 & 4283 & 5176 \\
\hline Morelos & 22404 & 39330 & 13017 & 26784 & 78195 & 191625 & 2408 & 3419 \\
\hline $\mathrm{N}$ ayarit & 28057 & 35397 & 16035 & 28207 & 46570 & 93146 & 5308 & 6342 \\
\hline Nuevo León & 72937 & 161626 & 61798 & 193730 & 108530 & 374726 & 6100 & 8310 \\
\hline 0 axaca & 41364 & 104160 & 34191 & 66151 & 88984 & 203847 & 7276 & 9091 \\
\hline Puebla & 79953 & 156969 & 67458 & 128943 & 137014 & 288448 & 9141 & 14266 \\
\hline Q uerétaro & 17117 & 28935 & 5875 & 14204 & 29553 & 79370 & 972 & 1505 \\
\hline Q uintana Roo & 8965 & 21311 & 2774 & 5285 & 26597 & 60096 & 595 & 569 \\
\hline San Luis Potosí & 47620 & 60479 & 23110 & 52860 & 44894 & 109852 & 3277 & 4674 \\
\hline Sinaloa & 46236 & 59920 & 10624 & 28179 & 55154 & 169958 & 7944 & 8354 \\
\hline Sonora & 29118 & 36622 & 22527 & 46577 & 47351 & 102709 & 5470 & 5525 \\
\hline Tabasco & 62923 & 90063 & 17047 & 39831 & 64879 & 198839 & 4845 & 6540 \\
\hline Tamaulipas & 45486 & 70653 & 20061 & 59499 & 59090 & 188596 & 5848 & 7618 \\
\hline Tlaxcala & 45129 & 72329 & 22819 & 56642 & 36775 & 96586 & 2998 & 3607 \\
\hline Veracruz & 79586 & 168084 & 44865 & 102174 & 145758 & 405849 & 26036 & 32580 \\
\hline Yucatán & 16425 & 30386 & 16456 & 37104 & 34725 & 94020 & 2146 & 2052 \\
\hline Zacatecas & 43997 & 77501 & 26779 & 63405 & 53093 & 142008 & 3664 & 4390 \\
\hline
\end{tabular}

EUM $=$ Estados Unidos Mexicanos

Fuente: SSA, D GEI, SISPA 